\title{
Effective Complexity and its Relation to Logical Depth
}

\author{
Nihat Ay, Markus Müller, Arleta Szkoła
}

\begin{abstract}
Effective complexity measures the information content of the regularities of an object. It has been introduced by $M$. Gell-Mann and S. Lloyd to avoid some of the disadvantages of Kolmogorov complexity, also known as algorithmic information content. In this paper, we give a precise formal definition of effective complexity and rigorous proofs of its basic properties. In particular, we show that incompressible binary strings are effectively simple, and we prove the existence of strings that have effective complexity close to their lengths. Furthermore, we show that effective complexity is related to Bennett's logical depth: If the effective complexity of a string $x$ exceeds a certain explicit threshold then that string must have astronomically large depth; otherwise, the depth can be arbitrarily small.
\end{abstract}

Index Terms-Effective Complexity, Kolmogorov Complexity, Algorithmic Information Content, Bennett's Logical Depth, Kolmogorov Minimal Sufficient Statistics, Shannon Entropy.

\section{INTRODUCTION AND MAIN RESULTS}

W HAT is complexity? A great deal of research has been performed on the question in what sense some objects are "more complicated" than others, and how this fact and its consequences can be analyzed mathematically.

One of the most well-known complexity measures is $\mathrm{Kol}$ mogorov complexity [1], also called algorithmic complexity or algorithmic information content. In short, the Kolmogorov complexity of some finite binary string $x$ is the length of the shortest computer program that produces $x$ on a universal computer. So Kolmogorov complexity quantifies how well a string can in principle be compressed. This notion of complexity has found various interesting applications in mathematics and computer science.

Despite its usefulness, Kolmogorov complexity does not capture the intuitive notion of complexity very well. For example, random strings without any regularities, say strings that are constructed bitwise by repeated tosses of a fair coin, have very large Kolmogorov complexity. But those strings are not "complex" from an intuitive point of view - those strings are completely random and do not carry any interesting structure at all.

Effective complexity is an attempt by M. Gell-Mann and S. Lloyd [2],[3] to define some complexity measure that is closer to the intuitive notion of complexity and overcomes the difficulties of Kolmogorov complexity. The main idea of effective complexity is to split the algorithmic information content of some string $x$ into two parts, its random features and

N. Ay, M. Müller and A. Szkoła are with the Max Planck Institute for Mathematics in the Sciences, Inselstr. 22, 04103 Leipzig, Germany. e-mail: \{nay,szkola\}@mis.mpg.de, mueller@math.tu-berlin.de. M. Müller is also with the Institute of Mathematics 7-2, TU Berlin, Straße des 17. Juni 136, 10623 Berlin, Germany. its regularities. Then, the effective complexity of $x$ is defined as the algorithmic information content of the regularities alone.

In this paper, we are interested in the basic properties of effective complexity, and how it relates to other complexity measures. In particular, we give a more precise formal definition of effective complexity than has been done previously. We use this formal framework to give detailed proofs of the properties of effective complexity, and we use it to show an unexpected relation between effective complexity and Bennett's logical depth [4].

Since there are now so many different complexity measures [5], our result contributes to the clarification of the interrelations within this "zoo" of complexity measures. Moreover, we hope that our more formal approach helps to find applications of effective complexity within mathematics, in a similar manner as this has been done for Kolmogorov complexity.

We now describe our main results and give a synopsis of this paper:

- After some notational preliminaries in Section [I, we motivate and state the main definition of effective complexity in Section III

- In Section IV we analyze the basic properties of effective complexity. In particular, we show in Theorem 10 that effective complexity indeed avoids the disadvantage of Kolmogorov complexity that we have explained above: Random strings are effectively simple.

Although the existence of effectively complex strings has been mentioned in [2], it has not been conjectured explicitly. Based on the notion of algorithmic statistics as studied by Gács at al. [6] we provide a formal existence proof, see Theorem 14.

- Section $\mathrm{V}$ contains our main result (Theorem 18 and Theorem [19), the relation between effective complexity and logical depth. In short, it states that if the effective complexity of some string exceeds a certain explicit threshold, then the time it takes to compute that string from a short description must be astronomically large. This threshold is in some sense very sharp, such that the behavior of logical depth with respect to effective complexity is comparable to that of a phase transition (cf. Fig. 2 on page 11).

- In Section VI we show how effective complexity is related to the notion of Kolmogorov minimal sufficient statistics.

- Finally, in the Appendix, we give an explicit example of a computable ensemble on the binary strings that has non-computable entropy. This illustrates the necessity of 
the details of our definition in Section [III

We start by introducing notation.

\section{Preliminaries AND Notation}

We denote the finite binary strings $\{\lambda, 0,1,00,01, \ldots\}$ by $\{0,1\}^{*}$, where $\lambda$ is the empty string, and we write $\ell(x)$ for the length of a binary string $x \in\{0,1\}^{*}$. An ensemble $\mathbb{E}$ is a probability distribution on $\{0,1\}^{*}$. All logarithms are in base 2 .

We assume that the reader is familiar with the basic concepts of Kolmogorov complexity; a good reference is the book by Li and Vitányi [1]. There is a "plain" and a "prefix" version of Kolmogorov complexity, and we will use both of them in this paper. The plain Kolmogorov complexity $C(x)$ of some string $x$ is defined as the length of the shortest computer program that outputs $x$ if it is given as input to a universal computer $V$,

$$
C(x):=\min \{\ell(p) \mid V(p)=x\} .
$$

Prefix Kolmogorov complexity is defined analogously, but with respect to a universal prefix computer $U$. A prefix computer $U$ has the property that if $U(s)$ is defined for some string $s$, then $U(s t)$ is undefined for every string $t$ that is not the empty string. So

$$
K(x):=\min \{\ell(p) \mid U(p)=x\} .
$$

There are different possible choices of $U$ and $V$; we fix one of them for the rest of the paper.

Several variations of Kolmogorov complexity can be easily defined and will be used in this paper, for example, the complexity of a finite list of strings, or the complexity of an integer or a real number. With a few exceptions below, we will not discuss the details of the definitions here and instead refer the reader to Ref. [1].

The first exception that deserves a more detailed discussion is conditional complexity. There are two versions of conditional complexity, a "naive" one and a more sophisticated one. The naive definition is

$$
K(x \mid y):=\min \left\{\ell(p) \mid p \in\{0,1\}^{*}, U(p, y)=x\right\},
$$

that is, the complexity of producing string $x$, given string $y$ as additional "free" information. A more sophisticated version due to Chaitin [7] reads

$$
K_{*}(x \mid y):=\min \left\{\ell(p) \mid p \in\{0,1\}^{*}, U\left(p, y^{*}\right)=x\right\},
$$

that is, the complexity of producing $x$, given a minimal program $y^{*}$ for $y$. The advantage of $K_{*}(\cdot \mid \cdot)$ compared with $K(\cdot \cdot \cdot)$ is the validity of a chain rule

$$
K(x, y) \stackrel{ \pm}{=} K(y)+K_{*}(x \mid y)
$$

for all strings $x$ and $y$. Here we make use of a wellknown notation [6] which helps to suppress additive constants: Suppose that $f, g:\{0,1\}^{*} \rightarrow \mathbb{N}$ are functions on the binary strings, and there is some $c \in \mathbb{N}$ independent of the argument value $s$ such that $f(s) \leq g(s)+c$ for every $s \in\{0,1\}^{*}$, i.e. the inequality holds uniformly for $s \in\{0,1\}^{*}$. Then we write

$$
f(s) \stackrel{+}{<} g(s) \quad\left(s \in\{0,1\}^{*}\right) .
$$

We use the notation $\stackrel{ \pm}{=}$ if both $\stackrel{+}{<}$ and $\stackrel{+}{>}$ hold.

Note that the "naive" form of conditional complexity as defined in (1) does not satisfy the chain rule (3). Only the weaker identity

$$
K(x, y) \stackrel{+}{<} K(y)+K(x \mid y)
$$

holds in general.

We will often use obvious identities like $K(x) \stackrel{+}{<} K(x, y)$ or $K(x, y) \stackrel{ \pm}{=} K(y, x)$ without explaining in detail where they come from; we again refer the reader to the book by $\mathrm{Li}$ and Vitányi [1].

Another important prerequisite for this paper is the definition of the prefix Kolmogorov complexity $K(\mathbb{E})$ of some ensemble $\mathbb{E}$. In contrast to bit strings, there are several inequivalent notions of a "description", and we can learn from Ref. [6] the lesson that it is very important to exactly specify which of them we will use.

Our definition of $K(\mathbb{E})$ for ensembles $\mathbb{E}$ is as follows. First, a program that computes $\mathbb{E}$ is a computer program that expects two inputs, namely a string $s \in\{0,1\}^{*}$ and an integer $n \in \mathbb{N}$, and that outputs (the binary digits of) an approximation of $\mathbb{E}(s)$ with accuracy of at least $2^{-n}$. Then, our preliminary definition of $K(\mathbb{E})$ is the length of the shortest program for the universal prefix computer $U$ that computes $\mathbb{E}$.

Obviously, not every ensemble $\mathbb{E}$ is computable - there is a continuum of string ensembles, but there are only countably many algorithms that compute ensembles. Another unexpected difficulty concerns the entropy of a computable ensemble, defined as $H(\mathbb{E}):=-\sum_{x \in\{0,1\}^{*}} \mathbb{E}(x) \log \mathbb{E}(x)$. Contrary to a first naive guess, the entropy of a computable ensemble does not need to be computable; all we know for sure is that it is enumerable from below. To illustrate this, we give an explicit example of a computable ensemble with a non-computable entropy in Example 22 in Appendix A.

Thus, for the rest of the paper, we assume that all ensembles are computable and have computable and finite entropy $H(\mathbb{E})$, unless stated otherwise.

Even when one restricts to the set of ensembles with computable entropy, the map $\mathbb{E} \mapsto H(\mathbb{E})$ is not necessarily a computable function. Hence the approximate equality

$$
K(\mathbb{E}, H(\mathbb{E})) \stackrel{ \pm}{=} K(\mathbb{E})
$$

is not necessarily true uniformly in $\mathbb{E}$. Thus, from now on we replace the preliminary definition $K(\mathbb{E})$ by

$$
K(\mathbb{E}):=K(\mathbb{E}, H(\mathbb{E})),
$$

i.e. we assume that computer programs for ensembles $\mathbb{E}$ carry additionally a subprogram that computes the entropy $H(\mathbb{E})$.

\section{Definition of EfFective Complexity}

To define the notion of effective complexity, we follow the steps described in one of the original manuscripts by M. GellMann and S. Lloyd [3]. First, they define the total information of an ensemble as the sum of the ensemble's entropy and complexity. 
To understand the motivation behind this definition, suppose we are given some data $x$ (a finite binary string) which has been generated by an unknown stochastic process. We would like to make a good guess on the process that generated $x$, even if we only have one sample of the process. This is similar to a scientist that tries to find a (probabilistic) theory of physics, given only the present state of the universe. To make a good guess on the probability distribution or ensemble $\mathbb{E}$ that produced $x$, we make two natural assumptions:

- The explanation should be simple. In terms of Kolmogorov complexity, this means that $K(\mathbb{E})$ should be small.

- The explanation should not allow all possible outcomes, but should prefer some outcomes (including $x$ ) over others. For example, the uniform distribution on a billion different possible physical theories is "simple" (i.e. $K(\mathbb{E})$ is small), but it is not a "good explanation" of our physical world because it contains a huge amount of arbitrariness. This arbitrariness can be identified with the "measure of ignorance", the entropy of $\mathbb{E}$. Thus, it is natural to demand that the entropy $H(\mathbb{E})$ shall be small.

Putting both assumptions together, it is natural to consider the sum $K(\mathbb{E})+H(\mathbb{E})$ which is called the "total information" $\Sigma(\mathbb{E})$. A "good theory" is then an ensemble $\mathbb{E}$ with small $\Sigma(\mathbb{E})$.

\section{Definition 1 (Total Information):}

For every ensemble $\mathbb{E}$ with entropy $H(\mathbb{E})$ := $-\sum_{x \in\{0,1\}^{*}} \mathbb{E}(x) \log \mathbb{E}(x)$, we define the total information $\Sigma(\mathbb{E})$ of $\mathbb{E}$ as

$$
\Sigma(\mathbb{E}):=K(\mathbb{E})+H(\mathbb{E}) .
$$

Note that the total information is a real number larger than or equal to 1 . If $\mathbb{E}$ is computable and has finite entropy, as always assumed in this paper, then $\Sigma(\mathbb{E})$ is finite.

In the subsequent work [2] by M. Gell-Mann and S. Lloyd, it has been pointed out that $H(\mathbb{E}) \approx \sum_{s \in\{0,1\}^{*}} \mathbb{E}(s) K(s \mid \mathbb{E})$. It follows that

$$
\Sigma(\mathbb{E}) \approx \sum_{s \in\{0,1\}^{*}} \mathbb{E}(s)(K(s \mid \mathbb{E})+K(\mathbb{E})) .
$$

This has a nice interpretation: The total information gives the average complexity of computing a string with the detour of computing the ensemble.

The next step in [3] is to explain what is meant by a string being "typical" for an ensemble. Going back to the analogy of a scientist trying to find a theory $\mathbb{E}$ explaining his data $x$, a good theory should in fact predict that the appearance of $x$ has non-zero probability. Even more, the probability $\mathbb{E}(x)$ should not be too small; it should be at least as large as that of "typical" outcomes of the corresponding process.

What is the probability of a "typical" outcome of a random experiment? Suppose we toss a biased coin with probability $p$ for heads and $1-p=: q$ for tails $n$ times, and call the resulting probability distribution $\mathbb{E}$. Then it turns out that typical outcomes $x$ have probability $\mathbb{E}(x)$ close to $2^{-n H}$, where $H:=-p \log p-q \log q$, and $n \cdot H$ is the entropy of $\mathbb{E}$. In fact, the probability that $\mathbb{E}(x)$ lies in between $2^{-n(H+\varepsilon)}$ and $2^{-n(H+\varepsilon)}$ for $\varepsilon>0$ tends to one as $n$ gets large. In information theory, this is called the "asymptotic equipartition property" (cf. Ref. [8]). An appropriately extended version of this result holds for a large class of stochastic processes, including ergodic processes.

This motivates to define that a string $x$ is typical for an ensemble $\mathbb{E}$ if its probability is not much smaller than $2^{-H(\mathbb{E})}$.

\section{Definition 2 ( $\delta$-Typical String):}

Let $\mathbb{E}$ be an ensemble, $x \in\{0,1\}^{*}$ a string and $\delta \geq 0$. We say that $x$ is $\delta$-typical for $\mathbb{E}$, if

$$
\mathbb{E}(x) \geq 2^{-H(\mathbb{E})(1+\delta)} .
$$

We return to the scenario of the scientist who looks for good theories (ensembles $\mathbb{E}$ ) explaining his data $x$. As discussed above, it is natural to look for theories with small total information $\Sigma(\mathbb{E})$. Moreover, the theory should predict $x$ as a "typical" outcome of the corresponding random experiment, that is, $x$ should be $\delta$-typical for $\mathbb{E}$ for some small constant $\delta$.

How small can the total information of such a theory be? The next lemma shows that the answer is "not too small".

Lemma 3 (Minimal Total Information):

It uniformly holds for $x \in\{0,1\}^{*}$ and $\delta \geq 0$ that

$$
\frac{K(x)}{1+\delta} \stackrel{+}{<} \inf \{\Sigma(\mathbb{E}) \mid x \text { is } \delta \text {-typical for } \mathbb{E}\} \stackrel{+}{<} K(x) .
$$

Remark. The upper bound $K(x)$ and the computability of $\mathbb{E}$ show that the set is finite, and the infimum is indeed a minimum.

Proof: Fix some $\delta \geq 0$ and some $x \in\{0,1\}^{*}$. Clearly, $x$ is $\delta$-typical for the singlet distribution $\mathbb{E}_{x}$, given by $\mathbb{E}_{x}(x)=1$ and $\mathbb{E}_{x}\left(x^{\prime}\right)=0$ for every $x^{\prime} \neq x$. This ensemble has entropy $H\left(\mathbb{E}_{x}\right)=0$. Thus, the total information $\Sigma\left(\mathbb{E}_{x}\right)$ equals the complexity $K\left(\mathbb{E}_{x}\right)$. We also have

$$
K\left(\mathbb{E}_{x}\right) \stackrel{ \pm}{=} K(x),
$$

as describing the ensemble $\mathbb{E}_{x}$ boils down to describing the string $x$. Furthermore, the corresponding additive constant does not depend on $x$ or $\delta$. It follows that $\inf \{\Sigma(\mathbb{E})\} \stackrel{+}{<} K(x)$.

To prove the converse, suppose $\mathbb{E}$ is any ensemble such that $x$ is $\delta$-typical for $\mathbb{E}$. Then we have the chain of inequalities

$$
\begin{aligned}
K(x) & \stackrel{+}{<}(x, \mathbb{E}) \\
& +K(\mathbb{E})+K(x \mid \mathbb{E}) \\
& +K(\mathbb{E})+\lceil-\log \mathbb{E}(x)\rceil \\
& \leq K(\mathbb{E})+\lceil H(\mathbb{E})(1+\delta)\rceil \\
& \leq \Sigma(\mathbb{E})+\delta H(\mathbb{E})+1 \\
& \leq \Sigma(\mathbb{E})(1+\delta)+1 .
\end{aligned}
$$

The first two inequalities follow from general properties of prefix Kolmogorov complexity, while the third inequality is due to the upper bound

$$
K(x \mid \mathbb{E}) \stackrel{+}{<}\lceil-\log \mathbb{E}(x)\rceil,
$$

which follows from coding every string $x$ with $\mathbb{E}(x) \neq 0$ into a prefix code word of length $\lceil-\log \mathbb{E}(x)\rceil$ (such a code exists due to the Kraft inequality). Moreover, (6) is a consequence 
of $\delta$-typicality of $s$ for $\mathbb{E}$, and (7) uses the definition of the total information $\Sigma$.

The ultimate goal of effective complexity is to assign a useful complexity measure $\mathcal{E}(x)$ to strings $x$. In our analogy, this means that the scientist wants to assign a natural number to his data $x$ saying how "complex" $x$ is. Simply taking the Kolmogorov complexity $K(x)$ as this value has important drawbacks: It does not at all capture the intuition that "complexity" should measure the "amount of structure" of an object. In fact, if $x$ is uniformly random (i.e. the result of fair coin tossing), then $K(x)$ is large, while the string possesses almost no structure at all.

The strategy of S. Lloyd and M. Gell-Mann [3] is instead to take that complexity $K(\mathbb{E})$ of "the best" theory $\mathbb{E}$ that explains the data $x$. What is "the best" theory? As already discussed, a good theory should have small total information $\Sigma(\mathbb{E})$, and the data $x$ should be "typical" for $\mathbb{E}$ in the sense that the probability $\mathbb{E}(x)$ is not much smaller than $2^{-H(\mathbb{E})}$.

Given some data $x$, there are always many "good theories" which satisfy these requirements. Which one is "the best"? To think about this question, it is helpful to look at a graphical representation of "good theories" and their properties as described in [3] and depicted in Fig. 1]

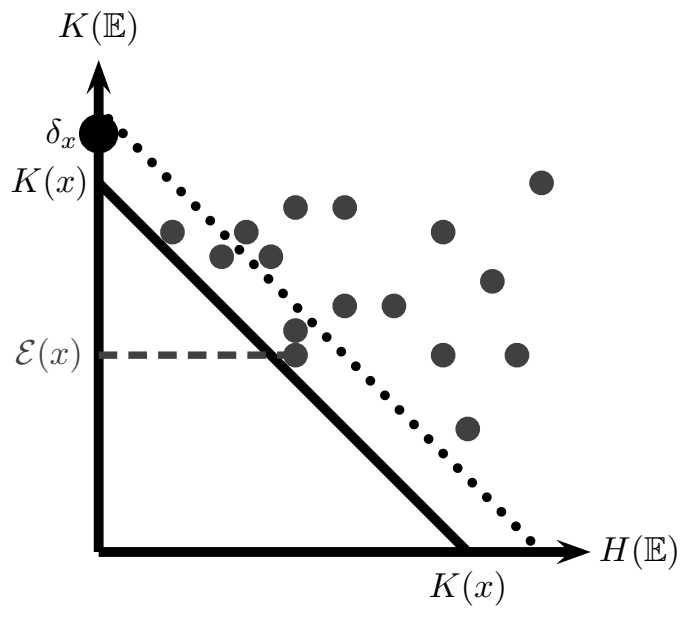

Fig. 1. The minimization domain of effective complexity. Plotted are only those ensembles $\mathbb{E}$ for which the fixed string $x$ is typical.

Suppose we plot the set of theories in the entropycomplexity plane. That is, for every computable ensemble $\mathbb{E}$ (with finite and computable entropy), we plot a black dot at the plane, where the $x$-axis labels the entropy $H(\mathbb{E})$ and the $y$-axis labels the Kolmogorov complexity $K(\mathbb{E})$.

The Kolmogorov complexity $K(\mathbb{E})$ is integer-valued, and if $n \in \mathbb{N}$ is small, there are only few ensembles $\mathbb{E}$ with $K(\mathbb{E})=$ $n$ (in fact, the number of such ensembles is upper-bounded by $2^{n}$ ). Thus, there are only few black dots at small values of the $y$-axis. Going up the $y$-axis, the number of ensembles and hence the density of the black dots increases.

The total information $\Sigma(\mathbb{E})$ is the sum of the ensemble's entropy and complexity. Thus, ensembles with constant total information correspond to lines in the plane that are parallel to the tilted line in Fig. 1
Suppose we fix some data $x$ and plot only those ensembles $\mathbb{E}$ such that $x$ is $\delta$-typical for $\mathbb{E}$ for some fixed constant $\delta \geq 0$. This was one of our two requirements that a "good theory" $\mathbb{E}$ for $x$ should fulfill. That is, we dismiss all the ensembles for which $x$ is not a typical realization.

Then Lemma 3 tells us that all the remaining ensembles must, up to an additive constant, have total information larger than $K(x) /(1+\delta)$. Graphically, this means that all those ensembles must approximately lie right of the straight line with $H(\mathbb{E})+K(\mathbb{E})=K(x)$. One of these ensembles is the Dirac measure $\delta_{x}$, the ensemble with $\delta_{x}(x)=1$ and $\delta_{x}\left(x^{\prime}\right)=0$ for $x \neq x^{\prime}$ : It has Kolmogorov complexity $K\left(\delta_{x}\right) \stackrel{ \pm}{=} K(x)$ and entropy $H\left(\delta_{x}\right)=0$, hence minimal total information. It corresponds to the circle on the $y$-axis at the left end of the line.

We also have discussed a second requirement for a "good theory": The total information should be as small as possible. According to Lemma 3, this means that $\Sigma(\mathbb{E})$ should not be much larger than the Kolmogorov complexity $K(x)$. We identify the "good" theories as those ensembles that are not too far away from the line in Fig. 1, say, we consider those ensembles as "good" that are below the dotted line with $\Sigma(\mathbb{E})=K(x)+\Delta$.

Among the remaining good theories, which one is "the best"? The convincing suggestion by M. Gell-Mann and S. Lloyd is that the best theory is the simplest theory; that is, the ensemble $\mathbb{E}$ with the minimal Kolmogorov complexity $K(\mathbb{E})$. The complexity $K(\mathbb{E})$ of this minimizing ensemble is then defined as the effective complexity of $x$.

In other words, the effective complexity of $x$ is defined as the smallest possible Kolmogorov complexity of any "good theory" (satisfying the two requirements) for $x$. This suggests the following preliminary definition (we discuss an important modification below):

Definition 4 (Effective Complexity I):

Given parameters $\delta, \Delta \geq 0$, the effective complexity $\mathcal{E}_{\delta, \Delta}(x)$ of any string $x \in\{0,1\}^{*}$ is defined as

$$
\begin{array}{r}
\mathcal{E}_{\delta, \Delta}(x):=\inf \{K(\mathbb{E}) \mid x \text { is } \delta \text {-typical for } \mathbb{E}, \\
\Sigma(\mathbb{E}) \leq K(x)+\Delta\},
\end{array}
$$

or as $\infty$ if this set is empty.

We refer to the set on the right-hand side as the minimization domain of $x$ for effective complexity, and denote it by $\mathcal{P}_{\delta, \Delta}(x)$. Thus

$$
\mathcal{E}_{\delta, \Delta}(x)=\min _{\mathbb{E} \in \mathcal{P}_{\delta, \Delta}(x)} K(\mathbb{E}) .
$$

Note that ensembles $\mathbb{E}$ of the minimization domain $\mathcal{P}_{\delta, \Delta}(x)$ of $x \in\{0,1\}^{*}$ satisfy

$$
\frac{K(x)}{1+\delta} \stackrel{+}{<} \Sigma(\mathbb{E}) \leq K(x)+\Delta .
$$

This notion of effective complexity is closely related to a quantity called "Kolmogorov minimal sufficient statistics". We explain this fact in more detail in Definition 20 and Lemma 21 in Section VI below.

As pointed out by M. Gell-Mann and S. Lloyd, it is often useful to extend this definition of effective complexity by 
imposing additional conditions ("constraints") on the ensembles that are allowed in the minimization domain. There are basically two intuitive reasons why this is useful. To understand those reasons, we go back to the scenario of a scientist looking for good theories to explain his data $x$. Recall the interpretation of the minimization domain of effective complexity as the set of "good theories" for $x$. Reasons for considering constraints on the ensembles are:

- Given the string $x$, there might be certain properties of $x$ that the scientist judges to be important. Those properties should be explained by the theories in the sense that the properties are not just simple random coincidences, but necessary or highly probable properties of each outcome of the corresponding process.

For example, suppose that a scientist wants to find good theories that explain the present state of our universe. In addition, that scientist finds it particularly important and interesting that the value of the fine structure constant is about $\frac{1}{137}$ and would like to find theories that explain why this constant is close to that value. Then, he will only accept ensembles $\mathbb{E}$ that have expectation value of this constant not too far away from $\frac{1}{137}$.

In terms of effective complexity, this scientist views the appearance of a fine structure constant of about $\frac{1}{137}$ as an important structural (non-random) property of $x$, the encoded state of our universe. Thus, he considers this property as part of the regularities of $x$. Effective complexity is the Kolmogorov complexity of the regularities of $x$, thus, this scientist tends to find a larger value of effective complexity than other scientists who consider the fine structure constant as unimportant and random.

- The scientist might have additional information on the process that actually created $x$. This situation is often encountered in thermodynamics. Suppose that $x$ encodes some microscopic properties of a gas in a container that a scientist has measured. In addition to these measurement results, the scientist typically also has information on several macroscopic observables like the temperature or the total energy in the box - at least, crude upper bounds are usually given by basic properties of the laboratory physics. Then, a "good theory" consistent with the actual physical process within the lab must obey the additional constraints given by the macroscopic observables.

In terms of effective complexity, the macroscopic observables respectively the additional information contributes to the regularities of $x$ and enlarges its effective complexity.

Definition 5 (Effective Complexity II):

Given parameters $\delta, \Delta \geq 0$, the effective complexity $\mathcal{E}_{\delta, \Delta}(x \mid \mathcal{C})$ of a string $x$ constrained to a subset $\mathcal{C}$ of all ensembles is

$$
\begin{array}{r}
\mathcal{E}_{\delta, \Delta}(x \mid \mathcal{C}):=\inf \{K(\mathbb{E}) \mid x \text { is } \delta \text {-typical for } \mathbb{E}, \mathbb{E} \in \mathcal{C}, \\
\Sigma(\mathbb{E}) \leq K(x)+\Delta\}
\end{array}
$$

or as $\infty$ if this set is empty. The set on the right-hand side is called the constrained minimization domain of $x$ for effective complexity and equals $\mathcal{P}_{\delta, \Delta}(x) \cap \mathcal{C}$ according to the notation of Definition 4. Thus, $\mathcal{E}_{\delta, \Delta}(x \mid \mathcal{C})=\min _{\mathbb{E} \in \mathcal{P}_{\delta, \Delta}(x) \cap \mathcal{C}} K(\mathbb{E})$.
Note that we allow that $\mathcal{C}$ depends on $x$. This makes it possible, for example, to introduce the constraint that $x$ is an element of an ensemble of strings that all have the same length: Just take $\mathcal{C}$ as the set of probability distributions on $\{0,1\}^{n}$, where $n=\ell(x)$.

In general restricting the set of ensembles will increase the value of effective complexity, i.e.

$$
\mathcal{C} \subseteq \mathcal{D} \Rightarrow \mathcal{E}_{\delta, \Delta}(x \mid \mathcal{C}) \geq \mathcal{E}_{\delta, \Delta}(x \mid \mathcal{D}) .
$$

This is in agreement with our intuition. Indeed such restrictions give a way to demand explicitly that some regularities of the considered string $x$ appear as a consequence of regularities of the generating process. As such, they contribute to the effective complexity.

If the constrained set $\mathcal{C}$ or the constant $\Delta$ are too small such that the (constrained) minimization domain $\mathcal{P}_{\delta, \Delta}(x) \cap \mathcal{C}$ is empty, then effective complexity is infinite, according to Definition 5. Furthermore note that

- $\mathcal{E}_{\delta, \Delta}(x \mid \mathcal{C})$ is decreasing in $\delta$ and $\Delta$,

- if $\mathcal{E}_{\delta, \Delta}(x \mid \mathcal{C})$ is finite and $x \in\{0,1\}^{n}$, then

$$
\mathcal{E}_{\delta, \Delta}(x \mid \mathcal{C}) \leq K(x)+\Delta \leq n+\mathcal{O}(\log n) .
$$

In many situations in physics, the constrained sets $\mathcal{C}$ appearing in the definition of $\mathcal{E}_{\delta, \Delta}(x \mid \mathcal{C})$ consist of those ensembles that have expectation values of observables within certain intervals. That is, we have real-valued functions $f_{i}$, the observables, and the set of ensembles $\mathcal{C}$ consists of those ensembles $\mathbb{E}$ with

$$
\sum_{x \in\{0,1\}^{*}} \mathbb{E}(x) f_{i}(x) \in I_{i},
$$

where the sets $I_{i} \subset \mathbb{R}$ are intervals or possibly fixed real numbers. Sometimes it even makes sense to allow different intervals $I_{i}(\mathbb{E})$ for different ensembles $\mathbb{E}$; say, the intervals may all be centered around the same fixed expectation value, but may have a width which grows with the standard deviation of $\mathbb{E}$ with respect to the observable $f_{i}$.

This is explored in more detail in the following example.

Example 6 (Constraints and Observables):

Fix some string $x \in\{0,1\}^{*}$. Let $M$ be an index set and $\left\{f_{i}\right\}_{i \in M}$ a family of real-valued constraint functions on $\{0,1\}^{*}$ (the observables). We would like to define a constrained set of ensembles $\mathcal{C}_{x}$ with the following property: $\mathcal{C}_{x}$ shall contain all those ensembles which have expectation values of the observables $\left\{f_{i}\right\}$ that are "not too far away from" the actual values $f_{i}(x)$ of the observables evaluated on the string $x$. This is done in the following way:

To each observable $f_{i}$ and ensemble $\mathbb{E}$, associate a corresponding interval $I_{i}(\mathbb{E}) \subset \mathbb{R}$. The choice of those intervals is somewhat arbitrary - we only demand that they contain the expectation values of the corresponding observables, i.e.

$$
\sum_{s \in\{0,1\}^{*}} \mathbb{E}(s) f_{i}(s) \in I_{i}(\mathbb{E}) \quad \text { for all } i \in M .
$$

Then define the constrained set of ensembles $\mathcal{C}_{x}$ by

$$
\mathcal{C}_{x}:=\left\{\mathbb{E} \mid f_{i}(x) \in I_{i}(\mathbb{E}) \text { for all } i \in M\right\},
$$


that is, $\mathcal{C}_{x}$ consists of those ensembles $\mathbb{E}$ such that the corresponding interval (centered around the corresponding expectation value) contains the "correct" value of the observable evaluated at $x$.

The corresponding effective complexity value

$$
x \mapsto \mathcal{E}_{\delta, \Delta}\left(x \mid \mathcal{C}_{x}\right)
$$

has a natural interpretation as the effective complexity of $x$ if the observable properties $f_{i}$ of $x$ are judged to be important (or are fixed as macroscopic observables). Compare the discussion before Definition 5 .

To illustrate the notation introduced in the previous example, we look at the situation when we would like to define the effective complexity of strings under the constraint of fixed length. That is, suppose that we consider the length $\ell(x)$ of our string $x$ as an important regularity - or that we have additional side information that the unknown random process generates strings of fixed length only. In this case, it makes sense to look at the effective complexity $\mathcal{E}_{\delta, \Delta}\left(x \mid \mathcal{C}_{x}\right)$, where

$$
\mathcal{C}_{x}:=\{\mathbb{E} \mid \ell(y) \neq \ell(x) \Rightarrow \mathbb{E}(y)=0\} .
$$

Example 7 (Fixed Length Constraint):

Consider the effective complexity notion $\mathcal{E}_{\delta, \Delta}\left(x \mid \mathcal{C}_{x}\right)$ as explained above. Instead of using Equation (12), we can also use the notation of Example 6. we have only one constraint, so the index set $M$ satisfies $\# M=1$, for example $M=\{1\}$.

Our observable $f_{1}$ is then the characteristic function on the strings of length $\ell(x)$, that is,

$$
f_{1}(s):= \begin{cases}1 & \text { if } \ell(s)=\ell(x) \\ 0 & \text { otherwise }\end{cases}
$$

To every ensemble $\mathbb{E}$, we associate an interval $I_{1}(\mathbb{E})$ which only consists of the single real number that equals the corresponding expectation value of $f_{1}$, i.e.

$I_{1}(\mathbb{E})=\left\{\sum_{s \in\{0,1\}^{*}} \mathbb{E}(s) f_{1}(s)\right\}=\left\{\sum_{\ell(s)=\ell(x)} \mathbb{E}(s)\right\} \subset \mathbb{R}$.

It is then easy to see that the set $\mathcal{C}_{x}$ defined in Example 6 above equals the set in Equation (12).

Due to linearity, the constrained sets $\mathcal{C}_{x}$ in Example 6 are always convex. This property, together with a computability condition, will be useful in the following.

Definition 8 (Convex and Decidable Constraints): A set $\mathcal{C}$ of ensembles on the binary strings is called

- convex, if for every finite set of ensembles $\left\{\mathbb{E}_{i}\right\}_{i} \subseteq \mathcal{C}$, every computable convex combination $\sum_{i} \lambda_{i} \mathbb{E}_{i}$ with $\lambda_{i} \in$ $(0,1)$ and $\sum_{i} \lambda_{i}=1$, is also in $\mathcal{C}$,

- decidable, if there exists some algorithm that, given some string $x \in\{0,1\}^{*}$ as input, decides in finite time whether the Dirac measure on $x$ is an element of $\mathcal{C}$ or not, that is, whether the measure

$$
\delta_{x}(t):= \begin{cases}1 & \text { if } t=x \\ 0 & \text { if } t \neq x,\end{cases}
$$

satisfies $\delta_{x} \in \mathcal{C}$ or not. In this sense, we may define $K(\mathcal{C})$ as the length of the shortest computer program that computes the corresponding decision function.
We proceed by analyzing some basic properties of effective complexity.

\section{BASIC Properties of EfFective Complexity}

We have remarked that the effective complexity $\mathcal{E}_{\delta, \Delta}(x \mid \mathcal{C})$ can be infinite, for example, if the constant $\Delta$ is too small or the constrained set of ensembles $\mathcal{C}$ is too restrictive such that the minimization domain satisfies $\mathcal{P}_{\delta, \Delta}(x) \cap \mathcal{C}=\emptyset$. Thus, we start by proving a simple sufficient condition that guarantees that effective complexity is finite.

Lemma 9 (Finiteness of Effective Complexity): There is a constant $m \in \mathbb{N}$ such that

$$
\mathcal{E}_{\delta, \Delta}(x \mid \mathcal{C}) \leq K(x)+\Delta<\infty
$$

for all strings $x$ with Dirac measure $\delta_{x} \in \mathcal{C}, \delta \geq 0$ and $\Delta \geq m$.

Proof: Due to (11), we only have to prove that $\mathcal{E}_{\delta, \Delta}(x \mid \mathcal{C})$ is finite. According to Definition 5, it remains to prove that

$$
\mathcal{P}_{\delta, \Delta}(x) \cap \mathcal{C} \neq \emptyset
$$

under the conditions given above, where $\mathcal{P}_{\delta, \Delta}(x)$ is the minimization domain of $x$. To this end, we show that $\delta_{x} \in \mathcal{P}_{\delta, \Delta}(x)$. This follows from

- $\delta_{x}(x)=1=2^{-H\left(\delta_{x}\right)(1+\delta)}$ for every $\delta \geq 0$, so $x$ is $\delta$-typical for $\delta_{x}$

- $\Sigma\left(\delta_{x}\right)=H\left(\delta_{x}\right)+K\left(\delta_{x}\right)=K\left(\delta_{x}\right) \leq K(x)+m$, where $m \in \mathbb{N}$ is a constant.

Our first result resembles the example on p. 51 in [3]. Suppose that we have a random binary string $x$ of length $n$, maybe a string which has been determined by tossing a fair coin $n$ times. The Kolmogorov complexity of such a string typically satisfies $K(x) \approx n$, that is, structureless random strings have maximal Kolmogorov complexity. This was one of reasons for S. Lloyd's and M. Gell-Mann's criticism of Kolmogorov complexity and for their attempt to define effective complexity as a more useful and intuitive complexity measure.

The following theorem proves that random strings indeed have small effective complexity, which supports the point of view that effective complexity measures only the complexity of the non-random structure of a string. Before we state that theorem, we have to explain in detail what we mean by a "random" string.

It is well-known that most strings are incompressible, which is what we mean by "random" at this point. In more detail, if $r \in \mathbb{N}$ is some fixed parameter, the number of strings $x$ of length $n$ with prefix complexity $K(x) \leq n+K(n)-r$ does not exceed $2^{n-r+\mathcal{O}(1)}$ (cf. [1] Thm. 3.3.1]). That is, most strings are incompressible in the sense that $K(x) \geq n+K(n)-r$. We call such strings $r$-incompressible.

Theorem 10 (Incompressible Strings are Simple): There exists some global constant $c \in \mathbb{N}$ such that

$$
\mathcal{E}_{\delta, \Delta}(x) \leq \log n+\mathcal{O}(\log \log n)
$$

for all $r$-incompressible strings $x$ of length $n, \delta \geq 0$ and $\Delta \geq r+c$. 
Moreover, if $\mathcal{C}$ is a convex and decidable constrained set of ensembles, then for all $r$-incompressible strings $x$ of length $n$ with the property that the Dirac measure $\delta_{x} \in \mathcal{C}$, we have

$$
\mathcal{E}_{\delta, \Delta}(x \mid \mathcal{C}) \leq \log n+\mathcal{O}(\log \log n)+K(\mathcal{C})
$$

whenever $\delta \geq 0$ and $\Delta \geq r+c+K(\mathcal{C})$. Note that the $\log \log n$ term does not depend on $\mathcal{C}$.

Proof: With a suitable choice of $c$, the first part of the theorem is a special case of the second part (with $\mathcal{C}:=$ the set of all ensembles), so it is sufficient to prove the second part.

Suppose that $\mathcal{C}$ and $x$ satisfy the conditions of the theorem. Let $\mathbb{E}_{x \mid \mathcal{C}}$ be the uniform distribution on

$$
M_{x \mid \mathcal{C}}:=\left\{t \in\{0,1\}^{*} \mid \ell(t)=\ell(x), \delta_{t} \in \mathcal{C}\right\} .
$$

Then $H\left(\mathbb{E}_{x \mid \mathcal{C}}\right)=\log \# M_{x \mid \mathcal{C}} \leq \ell(x)$, and $\mathbb{E}_{x \mid \mathcal{C}}(x)=$ $\frac{1}{\# M_{x \mid \mathcal{C}}}=2^{-H\left(\mathbb{E}_{x \mid \mathcal{C}}\right)}$, so $x$ is $\delta$-typical for $\mathbb{E}_{x \mid \mathcal{C}}$. Moreover,

$$
K\left(\mathbb{E}_{x \mid \mathcal{C}}\right) \stackrel{+}{<} K(\ell(x))+K(\mathcal{C})
$$

Thus, $\Sigma\left(\mathbb{E}_{x \mid \mathcal{C}}\right) \stackrel{+}{<} \ell(x)+K(\ell(x))+K(\mathcal{C})$. The strings $x$ which are $r$-incompressible satisfy by definition $K(x) \geq$ $\ell(x)+K(\ell(x))-r$. Denoting by $r(x)$ the corresponding degree of incompressibility of $x$ gives

$$
\Sigma\left(\mathbb{E}_{x \mid \mathcal{C}}\right) \stackrel{+}{<} K(x)+r(x)+K(\mathcal{C}),
$$

i.e. there is a global constant $c \in \mathbb{N}$ such that $\Sigma\left(\mathbb{E}_{x \mid \mathcal{C}}\right) \leq$ $K(x)+r(x)+K(\mathcal{C})+c$. Now if $\Delta \geq r(x)+K(\mathcal{C})+c$, it follows from Definition 4 and $\mathbb{E}_{x \mid \mathcal{C}} \in \mathcal{C}$ that

$$
\begin{aligned}
\mathcal{E}_{\delta, \Delta}(x \mid \mathcal{C}) & \leq K\left(\mathbb{E}_{x \mid \mathcal{C}}\right) \leq K(\ell(x))+K(\mathcal{C})+\mathcal{O}(1) \\
& \leq \log \ell(x)+\mathcal{O}(\log \log \ell(x))+K(\mathcal{C}) .
\end{aligned}
$$

Note that according to the theorem above, every string $x \in$ $\{0,1\}^{*}$ becomes effectively simple if $\Delta$ is large enough. Indeed, for every $\delta \geq 0$, relation (13) is satisfied by $x$ if $\Delta$ is larger than the $x$-dependent threshold $\Delta_{\max }(x):=$ $\ell(x)+\log \ell(x)+c-K(x)$. (Here $c$ is the global constant appearing in Theorem 10.)

For strings $x$ of fixed length $n$, one can give an $n$-dependent threshold $\Delta_{\max }(n):=n+c$ such that $\mathcal{E}_{\delta, \Delta}(x) \stackrel{+}{<} \log n+$ $O(\log \log n)$ if $\Delta \geq \Delta_{\max }(n)$. On the other hand, due to Lemma 9 , to ensure $\mathcal{E}_{\delta, \Delta}(x)<\infty$ for $x \in\{0,1\}^{*}$, one should choose $\Delta$ not too small, namely $\Delta \geq m$, where $m$ was a global constant depending on the reference universal computer only.

These simple observations show that a discussion of the dependence of effective complexity for arbitrary but fixed strings $x \in\{0,1\}^{*}$ on the parameter $\Delta$ should be useful for a deeper understanding of the concept.

In a forthcoming paper [9], we investigate in more detail the behavior of effective complexity of long strings generated by stochastic processes for different choices of $\Delta=\Delta(n)$. For the rest of this paper, we assume $\Delta$ to be a fixed constant (not depending on $n$ or $x$ ) that is larger than the aforementioned constant $m$.

In what follows we strengthen the result in Theorem 10 in a way that will be interesting later in Section $\nabla$
Corollary 11: There exists some global constant $c \in \mathbb{N}$ such that uniformly

$$
\mathcal{E}_{\delta, \Delta}(x) \stackrel{+}{<} K(C(x))+r
$$

for all $r$-incompressible strings $x, \delta \geq 0$ and $\Delta \geq r+c$.

If $\mathcal{C}$ is a convex and decidable set of ensembles, then for all $x$ that additionally satisfy $\delta_{x} \in \mathcal{C}$ we have

$$
\mathcal{E}_{\delta, \Delta}(x \mid \mathcal{C}) \stackrel{+}{<} K(C(x))+r+K(\mathcal{C})
$$

whenever $\Delta \geq r+c+K(\mathcal{C})$.

Proof: It follows from the proof of Theorem 10 that

$$
\mathcal{E}_{\delta, \Delta}(x \mid \mathcal{C}) \stackrel{+}{<} K(\ell(x))+K(\mathcal{C}) .
$$

According to the definition of $r$-incompressibility, we also have $K(\ell(x)) \leq K(x)-\ell(x)+r$, thus $\mathcal{E}_{\delta, \Delta}(x \mid \mathcal{C}) \stackrel{+}{<} K(x)-$ $\ell(x)+r+K(\mathcal{C})$. Moreover, it holds [1] $K(x) \stackrel{+}{<} C(x)+$ $K(C(x))$ and $C(x) \stackrel{+}{<} \ell(x)$.

The fact that incompressible strings have small effective complexity - and most strings are incompressible - raises the question if there exists any string with large effective complexity at all. Fortunately, the answer is "yes"; otherwise, the notion of effective complexity would be an empty concept. On the one hand, we might drop a requirement posed in Theorem 10 There, we restricted to constrained sets $\mathcal{C}$ of ensembles that contain the Dirac measure $\delta_{x}$, which basically means that the string $x$ should itself fulfill the constraints that are used to define $\mathcal{C}$. The effective complexity of strings $x$ that do not meet this requirement might possibly be large.

On the other hand, even among strings that fulfill this requirement, there are still strings that are effectively complex, namely those strings that are called "non-stochastic" in the context of algorithmic and Kolmogorov minimal sufficient statistics [6]. Suppose we have a finite subset $S \subset\{0,1\}^{*}$ of the finite binary strings. There are elements $x \in S$ that are easy to specify, once the set $S$ is given, in the sense that $K(x \mid S)$ is small. For example, the smallest element of $S$ in lexicographical order has very small conditional complexity $K(x \mid S)$. We call such elements atypical. On the other hand, most of the elements of $S$ will not be special in such a way, such that we can specify them only by giving their "index" within the set $S$, which takes about $\log \# S$ bits. Thus, most elements $x \in S$ will have

$$
K(x \mid S) \stackrel{+}{>} \log \# S .
$$

We can call such elements typical for $S$. There is a lemma by Gács, Tromp, and Vitányi [6], stating that there exist strings which are atypical for every simple set $S$. They are called non-stochastic:

Lemma 12 ([6. Thm.IV.2]): There are constants $c_{1}, c_{2} \in \mathbb{N}$ such that the following holds true: Suppose $n \in \mathbb{N}$ is fixed. For every $k<n$, there is some string $x \in\{0,1\}^{n}$ with $K(x \mid n) \leq$ $k$, such that

$$
\log \# S-K(x \mid S, K(S))>n-k-c_{2}
$$

for every $S \ni x$ with $K(S)<k-c_{1}$. 
We want to use this result to prove that for every $n$, there are binary strings of length $n$ that have effective complexity of about $n$. Therefore, we have to show that basically all we do with ensembles of strings can as well be accomplished with equidistributions on sets:

Lemma 13 (Ensembles and Sets):

Let $x \in\{0,1\}^{*}$ and $\delta, \Delta \geq 0$ be arbitrary, and let $\mathcal{C}$ be a set of decidable and convex constraints such that $\delta_{x} \in \mathcal{C}$. Moreover, let $\mathcal{D}$ be an arbitrary set of constraints such that the effective complexity $\mathcal{E}_{\delta, \Delta}(x \mid \mathcal{D})$ is finite, and let $\mathbb{E}$ be the corresponding minimizing ensemble. Then, for every $\varepsilon>0$, there is a set $S \subset\{0,1\}^{*}$ containing $x$ with

$$
\begin{aligned}
\log \# S & \leq H(\mathbb{E})(1+\delta)+\varepsilon \\
K(S) & \leq K(\mathbb{E})+c+K(\delta)+K(\varepsilon)+K(\mathcal{C})
\end{aligned}
$$

such that the equidistribution on $S$ is in $\mathcal{C}$, where $c \in \mathbb{N}$ is a global constant.

Remark. The most interesting case is $\mathcal{D}=\mathcal{C}$, showing that the minimizing ensembles in the definition of effective complexity can "almost" (up to the additive terms above) be chosen to be equidistributions on sets even in the presence of decidable and convex constraints.

Proof: The minimizing ensemble $\mathbb{E}$ in the definition of $\mathcal{E}_{\delta, \Delta}(x \mid \mathcal{D})$ has the following properties:

$$
\begin{aligned}
\mathbb{E}(x) & \geq 2^{-H(\mathbb{E})(1+\delta),}, \\
K(\mathbb{E})+H(\mathbb{E}) & \leq K(x)+\Delta .
\end{aligned}
$$

We would like to write a computer program that, given a description of $\mathbb{E}$, computes a list of all strings $y \in\{0,1\}^{*}$ that satisfy the constraints $\mathcal{C}$ and the inequality $\mathbb{E}(y) \geq$ $2^{-H(\mathbb{E})(1+\delta)}$. Such a program could search through all strings $y$, decide for every $y$ whether this equation and the constraints hold for $y$, and do this until the sum of the probabilities of all the previously listed elements exceeds $1-2^{-H(\mathbb{E})(1+\delta)}$. But there is a problem of numerics: It is in general impossible for the program to decide with certainty if this inequality holds, because of the unavoidable numerical error. Instead, we can construct a computer program that computes a set $S \subset\{0,1\}^{*}$ with the following weaker properties:

$$
\begin{array}{r}
y \in S \Rightarrow \mathbb{E}(y) \geq 2^{-H(\mathbb{E})(1+\delta)-\varepsilon} \text { and } y \text { satisfies } \mathcal{C}, \\
\mathbb{E}(y) \geq 2^{-H(\mathbb{E})(1+\delta)} \text { and } y \text { satisfies } \mathcal{C} \Rightarrow y \in S .
\end{array}
$$

That is, the program computes a set $S$ which definitely contains all strings $y$ with $\mathbb{E}(y) \geq 2^{-H(\mathbb{E})(1+\delta)}$ that satisfy the constraints, but it may also contain strings which slightly violate this inequality as long as they still satisfy the constraints. However, the numerical methods are chosen good enough such that we are guaranteed that every element of $S$ has probability of at least $2^{-H(\mathbb{E})(1+\delta)-\varepsilon}$.

This set $S$ contains $x$ and has the desired properties (14) and (15). This can be seen as follows. By definition, $\mathbb{E}(x) \geq$ $2^{-H(\mathbb{E})(1+\delta)}$, so $x \in S$. Since every element $y \in S$ has probability $\mathbb{E}(y) \geq 2^{-H(\mathbb{E})(1+\delta)-\varepsilon}$, it holds $\# S \leq 2^{H(\mathbb{E})(1+\delta)+\varepsilon}$. Finally, the description length of the corresponding computer program can be estimated via $K(S \mid \mathbb{E}) \leq c+K(\delta)+K(\varepsilon)+$ $K(\mathcal{C})$.
Now we are ready to prove the existence of effectively complex strings. First, what should we expect from "effectively complex" strings - how large could effective complexity $\mathcal{E}_{\delta, \Delta}(x)$ of some string $x$ of length $n$ possibly be? If $\mathbb{E}$ is the minimizing ensemble in the definition of $\mathcal{E}_{\delta, \Delta}(x)$, then

$\mathcal{E}_{\delta, \Delta}(x)=K(\mathbb{E}) \leq \Sigma(\mathbb{E}) \leq K(x)+\Delta \leq n+K(n)+\mathcal{O}(1)$.

Thus, the best result we can hope for is the existence of strings of length $n$ that have effective complexity close to $n$. The next theorem shows exactly this.

Theorem 14 (Effectively Complex Strings):

For every $\delta, \Delta \geq 0$ and $n \in \mathbb{N}$, there is a string $x$ of length $n$ such that

$$
\mathcal{E}_{\delta, \Delta}(x) \geq(1-\delta) n-(1+2 \delta) \log n-\mathcal{O}(\log \log n) .
$$

As effective complexity is increased if constraints are added, the same statement is true for $\mathcal{E}_{\delta, \Delta}(x \mid \mathcal{C})$ if $\mathcal{C}$ is an arbitrary constrained set of ensembles.

Remark. An explicit lower bound is

$$
\begin{aligned}
\mathcal{E}_{\delta, \Delta}(x) \geq & (1-\delta) n-(1+2 \delta) \log n-2 \log \log n \\
& -\Delta(4+\delta)-5 K(\delta)-\omega
\end{aligned}
$$

where $\omega \in \mathbb{N}$ is a global constant.

Proof: Fix $\Delta \geq 0, \delta \in(0,1)$, and $x \in\{0,1\}^{n}$. Let $\mathbb{E}_{x}$ be the minimizing ensemble associated to $\mathcal{E}_{\delta, \Delta}(x)$, i.e.

$$
K\left(\mathbb{E}_{x}\right)=\mathcal{E}_{\delta, \Delta}(x) .
$$

Choose $\varepsilon>0$ arbitrary. According to Lemma 13, there is a set $S_{x} \subset\{0,1\}^{*}$ such that $x \in S_{x}$ and

$$
\begin{aligned}
\log \# S_{x} & \leq H\left(\mathbb{E}_{x}\right)(1+\delta)+\varepsilon, \\
K\left(S_{x}\right) & \leq K\left(\mathbb{E}_{x}\right)+c,
\end{aligned}
$$

where $c \in \mathbb{N}$ the sum of a global constant and $K(\delta)$ and $K(\varepsilon)$. Let $\tilde{c}$ be the best constant for our universal computer $U$ such that

$$
K(s) \leq K(s \mid t)+K(t)+\tilde{c} \quad \text { for all } s, t \in\{0,1\}^{*}
$$

and at the same time

$$
K(s \mid t) \leq K(s \mid t, u)+K(u)+\tilde{c} \quad \text { for all } s, t, u \in\{0,1\}^{*} .
$$

Using (16), (18) and (19), we conclude that $x$ is almost typical for $S_{x}$ :

$$
\begin{aligned}
K\left(x \mid S_{x}\right) \geq & K(x)-K\left(S_{x}\right)-\tilde{c} \\
\geq & K\left(\mathbb{E}_{x}\right)+H\left(\mathbb{E}_{x}\right)-\Delta-K\left(S_{x}\right)-\tilde{c} \\
\geq & K\left(S_{x}\right)-c+\frac{\log \# S_{x}}{1+\delta}-\frac{\varepsilon}{1+\delta}-\Delta \\
& -K\left(S_{x}\right)-\tilde{c} \\
\geq & \frac{\log \# S_{x}}{1+\delta}-c-\varepsilon-\Delta-\tilde{c} .
\end{aligned}
$$

It also follows

$$
\begin{aligned}
K\left(x \mid S_{x}, K\left(S_{x}\right)\right) \geq & K\left(x \mid S_{x}\right)-K\left(K\left(S_{x}\right)\right)-\tilde{c} \\
\geq & \frac{\log \# S_{x}}{1+\delta}-K\left(K\left(S_{x}\right)\right)-c-\varepsilon \\
& -\Delta-2 \tilde{c} .
\end{aligned}
$$


Now we get rid of the term $K\left(K\left(S_{x}\right)\right)$. First note that (19), (17) and (11) yield

$$
\begin{aligned}
K\left(S_{x}\right) & \leq K\left(\mathbb{E}_{x}\right)+c=\mathcal{E}_{\delta, \Delta}(x)+c \\
& \leq K(x)+\Delta+c \\
& \leq n+2 \log n+\gamma+\Delta+c,
\end{aligned}
$$

where $\gamma \in \mathbb{N}$ is some constant such that $K(s) \leq \ell(s)+$ $2 \log \ell(s)+\gamma$ for every $s \in\{0,1\}^{*}$, and $K(k) \leq \log k+$ $2 \log \log k+\gamma$ for every $k \in \mathbb{N}$. By elementary analysis, it holds $\log (a+b) \leq \log a+\frac{b}{a}$ if $a, b>0$. Hence there is some constant $\kappa>0$ which does not depend on $n, \delta, \Delta$, or $x$, such that for $n \geq 2$,

$$
\log K\left(S_{x}\right) \leq \log n+c+\Delta+\kappa .
$$

Using the same argument with $K\left(K\left(S_{x}\right)\right) \leq \log K\left(S_{x}\right)+$ $2 \log \log K\left(S_{x}\right)+\gamma$, for get for all $n \geq 2$

$$
K\left(K\left(S_{x}\right)\right) \leq \log n+2 \log \log n+3 c+3 \Delta+3 \kappa+\gamma .
$$

Going back to (20), it follows

$$
K\left(x \mid S_{x}, K\left(S_{x}\right)\right) \geq \frac{\log \# S_{x}}{1+\delta}-\log n-2 \log \log n-\Lambda,
$$

where

$$
\Lambda:=4 c+4 \Delta+3 \kappa+\gamma+\varepsilon+2 \tilde{c}
$$

Note that $x$ was arbitrary, so this equation is valid for every $x \in\{0,1\}^{n}$.

Let now $K_{n}:=\max \left\{K(t) \mid t \in\{0,1\}^{n}\right\}$, and

$$
k:=n-\left\lceil\delta\left(K_{n}+\Delta+\varepsilon\right)+\log n+2 \log \log n\right\rceil-\Lambda-c_{2},
$$

where $c_{2}$ is the constant from Lemma 12. If $n$ is large enough, then $0<k<n$ holds, and Lemma12 applies: There is a string $x^{*} \in\{0,1\}^{n}$ such that

$$
K\left(x^{*} \mid S, K(S)\right)<\log \# S-n+k+c_{2}
$$

for every set $S \ni x^{*}$ with $K(S)<k-c_{1}$, where $c_{1}$ is another global constant. First note the following inequality:

$$
\begin{aligned}
-\delta\left(K_{n}+\Delta+\varepsilon\right) & \leq-\delta\left(K\left(x^{*}\right)+\Delta+\varepsilon\right) \\
& \leq-\delta\left(H\left(\mathbb{E}_{x^{*}}\right)+\varepsilon\right) \\
& \leq-\delta\left(H\left(\mathbb{E}_{x^{*}}\right)+\frac{\varepsilon}{1+\delta}\right) \\
& =\frac{-\delta}{1+\delta}\left(H\left(\mathbb{E}_{x^{*}}\right)(1+\delta)+\varepsilon\right) \\
& \leq\left(\frac{1}{1-\delta}-1\right) \log \# S_{x^{*}} .
\end{aligned}
$$

Now suppose that $K\left(S_{x^{*}}\right)<k-c_{1}$. Consequently,

$$
\begin{aligned}
K\left(x^{*} \mid S_{x^{*}}, K\left(S_{x^{*}}\right)\right)< & \log \# S_{x^{*}}-n+k+c_{2} \\
\leq & \log \# S_{x^{*}}-n-\Lambda+n-\log n \\
& -\delta\left(K_{n}+\Delta+\varepsilon\right)-2 \log \log n \\
\leq & \frac{\log \# S_{x^{*}}}{1-\delta}-\log n-2 \log \log n-\Lambda .
\end{aligned}
$$

But this is a contradiction to (21). Hence our assumption must be false, and we must instead have $K\left(S_{x^{*}}\right) \geq k-c_{1}$. Thus, using (17), (19), and $K_{n} \leq n+2 \log n+\gamma$,

$$
\begin{aligned}
\mathcal{E}_{\delta, \Delta}\left(x^{*}\right)= & K\left(\mathbb{E}_{x^{*}}\right) \geq K\left(S_{x^{*}}\right)-c \\
\geq & k-c_{1}-c \\
\geq & n-\delta(n+2 \log n+\gamma+\Delta+\varepsilon)-\log n \\
& -2 \log \log n-\Lambda-c_{2}-1 .
\end{aligned}
$$

Applying relation (11) to the case of effectively complex strings $x^{*}$ constructed here, we obtain a lower bound on $K\left(x^{*}\right)$ :

$$
(1-\delta) n-(1+2 \delta) \log n-2 \log \log n-\theta \leq K\left(x^{*}\right),
$$

where $\theta=\Delta(5+\delta)+5 K(\delta)+\omega$. For large $n$, where the constant $\theta$ becomes negligible, this bound is non-trivial and in particular for $\delta=0$ remarkably close to the maximal value $n+K(n)$.

On the other hand, from the previous proof and Lemma 12 we deduce the following upper bound on the complexity of $x^{*}$ :

$K\left(x^{*}\right) \leq(1-\delta) n+K(n)-\log n-2 \log \log n-\Lambda+\tilde{\omega}$,

where $\tilde{\omega}$ is a global constant. This implies the following relation between the degree of incompressibility $r\left(x^{*}\right)$ and the constant $\Delta$ :

$$
r\left(x^{*}\right) \geq \delta n+\log n+2 \log \log n+\Lambda-\tilde{\omega} \geq \Lambda \geq 4 \Delta,
$$

where the second inequality holds for sufficiently large $n$ and the last one uses the definition (22) of $\Lambda$. Indeed, this relation prevents $x^{*}$ from falling into the domain of Theorem 10, which would force it to have small effective complexity.

Note that all effectively complex strings must, as long as effective complexity is finite, have large Kolmogorov complexity, too. This follows from (11).

\section{Effective Complexity and Logical Depth}

In this section, we show that effectively complex strings have very large computation times. In more detail, it takes a universal computer an astronomically large amount of time to compute such a string from its minimal program, or from an almost minimal program.

The time it takes to compute a string from its minimal program is discussed by C. Bennett [4] in the context of "logical depth". The notion of logical depth formalizes the idea that some strings are more difficult to construct than others (say, a string describing a proof of the Riemann hypothesis is harder to construct than a uniformly random string). However, the computation time of a string's minimal program is not a very stable measure for this difficulty: There might be programs that are "almost minimal", i.e. only a few bits longer, but run much faster than the minimal program.

Thus, logical depth is defined as the shortest time to compute a string from its almost-minimal program. "Almostminimal" means that the program is only a few bits longer than the minimal program, and the maximum length overhead is called the "significance level". 
Definition 15 ([4], Depth of Finite Strings):

Let $x \in\{0,1\}^{*}$ be a string and $z \in \mathbb{N}_{0}$ a parameter. A string's logical depth at significance level $z$, denoted $\operatorname{Depth}_{z}(x)$, will be defined as

$$
\operatorname{Depth}_{z}(x):=\min \{T(p) \mid \ell(p)-C(x) \leq z, V(p)=x\},
$$

where $T(p)$ denotes the halting time of the universal computer $V$ on input $p$.

Note that we have used plain Kolmogorov complexity $C$ here, that is, the universal computer $V$ is not assumed to be prefix-free in contrast to the original definition [4]. As plain and prefix Kolmogorov complexity are closely related, this modification will not result in large quantitative changes. However, for us it has important technical advantages as we will see below.

Logical depth is sometimes defined in a different manner with reference to algorithmic probability, cf. [1, Def. 7.7.1]. However, if computation times are large (which will be the case in our context, cf. the remark after Theorem [18), then those different definitions are essentially equivalent [1, Claim 7.7.1].

Clearly, it takes a computer (i.e. a Turing machine) at least $\ell(x)$ steps to print a string $x$ on its tape. Thus, the depth of a string must be lower-bounded by its length: $\operatorname{Depth}_{z}(x) \stackrel{+}{>} \ell(x)$ for every $z$. Following [1], strings that have a depth almost as small as possible, i.e. $\operatorname{Depth}_{z}(x) \stackrel{+}{<} \ell(x)$, will be called shallow.

The notion of depth depends on the choice of the universal reference computer - but not too much. As explained in [1], there is a universal 2-tape Turing machine that simulates $t$ steps of an arbitrary $k$-tape Turing machine in time $c \cdot t \log t$, where $c$ is a constant that only depends on the machine. In particular, this Turing machine can implement obvious tasks like copying of $n$ bits in time $n$ from one tape to the other. Therefore, we will fix this "Hennie-Sterns machine" (cf. [1. 6.13]) as our universal reference machine for this section.

To state the first example, a string $x$ will be called $m$ random if $C(x) \geq \ell(x)-m$.

Example 16 ([] Ex. 7.7.3]): Random strings are shallow. That is, there are constants $\beta, \gamma \in \mathbb{N}$ such that for every $m$ random string $x$ it holds

$$
\operatorname{Depth}_{m+\beta}(x) \leq \ell(x)+\gamma \text {. }
$$

As Depth $_{z}$ is decreasing in $z$, it also follows that $\operatorname{Depth}_{z}(x) \leq \ell(x)+\gamma$ for all $z \geq m+\beta$.

Proof: There is always a computer program $p$ of length $\ell(x)+\beta$ that sequentially lists the bits of $x$, producing $V(p)=$ $x$ in the most trivial way. Such a program will have a running time of $\ell(x)$, plus potentially some overhead $\gamma$ resulting from initialization. If $\ell(x) \leq C(x)+m$, then $\ell(p) \leq C(x)+m+\beta$, and the claim follows from the definition of depth.

It will be interesting in the following that this conclusion carries over to "most" strings that are $r$-incompressible as defined in Theorem 10. This will be proved in the next example. Moreover, we give a technical result which will be useful below. Note that

$$
K(x) \stackrel{+}{<} C(x)+K(C(x)) \quad\left(x \in\{0,1\}^{*}\right),
$$

and we will be interested in strings for which some kind of converse holds. For this purpose, we say that a string $x$ is $k$-well-behaved for some $k \in \mathbb{N}$ if

$$
K(x)+k \geq C(x)+K(C(x)) .
$$

In fact, it is stated in [1] that most strings are $k$-well-behaved if $k$ is large enough. The next example shows that incompressible random strings are well-behaved.

Lemma 17 (Incompressible and Shallow Strings): For every $n$, there are at least $2^{n}\left(1-2^{-r+c}-2^{-m}\right)$ strings of length $n$ that are $r$-incompressible and $m$-random, where $c \in \mathbb{N}$ is a constant. They satisfy

$$
\operatorname{Depth}_{m+\beta}(x) \leq \ell(x)+\gamma,
$$

where $\beta, \gamma \in \mathbb{N}$ are constants. Moreover, those strings are $k$ well-behaved, where $k \in \mathbb{N}$ is a constant that only depends on $r$ and $m$, but not on $x$.

Proof: Recall two basic incompressibility facts that are listed in [1]:

- There is a constant $c$ such that there are at least $2^{n}(1-$ $2^{-r+c}$ ) strings of length $n$ which are $r$-incompressible.

- For every $m \in \mathbb{N}$, there are at least $2^{n}\left(1-2^{-m}\right)$ strings of length $n$ with $C(x) \geq n-m$ (we call such strings " $m$-random").

A simple application of the union bound gives that there are at least $2^{n}\left(1-2^{-r+c}-2^{-m}\right)$ strings of length $n$ that are at the same time $r$-incompressible and $m$-random. The upper bound on the logical depth follows then from Example 16

If $x$ is $m$-random, then $C(x) \geq \ell(x)-m$, and a converse inequality holds trivially. The well-known continuity property [1] of $K$ then guarantees the existence of a constant $l \in \mathbb{N}$ (that depends only on $m$ but not on $x$ ) such that $K(C(x)) \leq K(\ell(x))+l$. Moreover, if $p \in \mathbb{N}$ is a constant such that $C(s) \leq \ell(s)+p$ for all strings $s$, the $r$-incompressibility property yields

$$
\begin{aligned}
K(x) & \geq \ell(x)+K(\ell(x))-r \\
& \geq C(x)-p+K(C(x))-l-r .
\end{aligned}
$$

It follows that $x$ is $(p+l+r)$-well-behaved.

We will now show that effectively complex strings must have very large logical depth if they are well-behaved. This is in contrast to the fact that incompressible strings (which have small effective complexity according to Theorem (10) are always shallow, that is, have very small depth as shown in Lemma 17

The main idea is as follows: Suppose that an almostminimal program for the string $x$ has a rather short halting time $\tau$. Then we can consider the ensemble $\mathbb{E}$, that is defined by equidistribution over all strings that have a short program (of length less or equal to $C(x)$ ) with some halting time less than or equal to $\tau$. Such an ensemble is simple, $x$ is typical for it, and it has small total information. Thus, it forces the effective complexity of $x$ to be small.

Theorem 18 (Effective Complexity and Depth): There is a global constant $\omega \in \mathbb{N}$ with the following property: Suppose that $f: \mathbb{N} \rightarrow \mathbb{N}$ is a strictly increasing, computable function. 
Furthermore, suppose that $x$ is a $k$-well-behaved string. If the effective complexity of $x$ satisfies

$$
\mathcal{E}_{\delta, k+z+K(z)+K(f)+\omega+1}(x)>K(C(x))+K(z)+K(f)+\omega
$$

for some arbitrary $\delta \geq 0$ and $z \in \mathbb{N}$, then

$$
\operatorname{Depth}_{z}(x)>f(C(x)) \text {. }
$$

Remark. Before we prove this theorem, we explain its meaning and implications. First, since $C(x) \stackrel{+}{<} \ell(x)$, it follows that $K(C(x))$ is of the order $\log \ell(x)$ or less, which is quite small. Thus, the inequality for $\mathcal{E}$ in this theorem is a very weak condition.

The function $f$ can be chosen to be simple (such that $K(f)$ is small), but extremely rapidly growing, for example of the form

$$
f(n):=n^{n^{n^{n \cdots}}} \text { (power tower of height } n \text { ). }
$$

Thus, the consequence of this theorem is that the depth must be astronomically large.

Proof: As $\mathcal{E}_{\delta, \Delta}$ is decreasing in $\delta$, it is sufficient to prove the theorem for the case $\delta=0$. For $y \in \mathbb{N}$ and every computable function $f: \mathbb{N} \rightarrow \mathbb{N}$, define the set

$$
\begin{aligned}
\tau_{y, f}:= & \left\{x \in\{0,1\}^{*} \mid \exists p \in\{0,1\}^{*} \text { with } V(p)=x,\right. \\
& \ell(p) \leq y, T(p) \leq f(y)\},
\end{aligned}
$$

where $T(p)$ denotes the halting time of the (plain, not prefix) universal computer $V$ on input $p$. Clearly $\# \tau_{y, f}<2^{y+1}$, and if $\mathbb{E}_{y, f}$ is the uniform distribution on $\tau_{y, f}$, then $H\left(\mathbb{E}_{y, f}\right)<y+1$. Moreover, there is some constant $c \in \mathbb{N}$ such that $K\left(\mathbb{E}_{y, f}\right) \leq$ $K(y)+K(f)+c$. Hence the total information satisfies

$$
\Sigma\left(\mathbb{E}_{y, f}\right) \leq y+K(y)+K(f)+c+1 .
$$

Now let $x \in\{0,1\}^{*}$ be a $k$-well behaved string, and let $z \in \mathbb{N}$ be arbitrary. Suppose that $x \in \tau_{C(x)+z, f}$, then $x$ is 0 -typical for $\mathbb{E}:=\mathbb{E}_{C(x)+z, f}$. Since there exists some global constant $d \in \mathbb{N}$ such that

$$
K(a+b) \leq K(a)+K(b)+d \text { for every } a, b \in \mathbb{N},
$$

we can estimate, using (25),

$$
\begin{aligned}
\Sigma(\mathbb{E}) & \leq C(x)+z+K(C(x)+z)+K(f)+c+1 \\
& \leq C(x)+K(C(x))+z+K(z)+K(f)+d+c+1 \\
& \leq K(x)+\underbrace{k+z+K(z)+K(f)+c+d+1}_{=: \Delta} .
\end{aligned}
$$

By definition of effective complexity, we get

$$
\begin{aligned}
\mathcal{E}_{0, \Delta}(x) & \leq K(\mathbb{E}) \leq K(C(x)+z)+K(f)+c \\
& \leq K(C(x))+K(z)+K(f)+c+d .
\end{aligned}
$$

In summary, we have so far shown the following: if there is a program $p \in\{0,1\}^{*}$ with $V(p)=c$ and $\ell(p) \leq C(x)+$ $z$ such that the corresponding halting time satisfies $T(p) \leq$ $f(C(x)+z)$, then the effective complexity is as small as in (26). The negation of this statement, together with $f(C(x)+$ $z) \geq f(C(x))$ and $\omega:=c+d$, proves the theorem.

Interestingly, incompressible strings just slightly fail to fulfill the inequality of the previous theorem. According to
Corollary 11, $r$-incompressible strings $x$ have effective complexity $\mathcal{E}_{\delta, r+c}(x) \stackrel{+}{<} K(C(x))+r$. Thus, we cannot conclude that those strings have large depth - fortunately, because most $r$-incompressible strings are in fact shallow according to Example 17.

Thus, it follows that the expression $K(C(x))$ sharply marks the "edge of depth", in the sense that strings with larger effective complexity always have extremely large depth, but strings with smaller effective complexity can have arbitrarily small depth. In some sense, a phenomenon similar to a "phase transition" occurs at effective complexity of $K(C(x)$ ) (apart from additive constants that get less and less important in the "thermodynamic limit" $\ell(x) \rightarrow \infty$ ).

This behavior is schematically depicted in Figure 2 The previous theorem says that if the effective complexity exceeds $K(C(x))$ (omitting all additive constants here), then the logical depth must be astronomically large. On the other hand, if effective complexity is smaller, different values of depth seem possible. In particular, if $x$ is $r$-incompressible, then we know from Corollary 11 that effective complexity is (possibly only up to a few bits) smaller than $K(C(x))$, while the logical depth is as small as possible (of the order $\ell(x)$ ) due to Lemma 17.

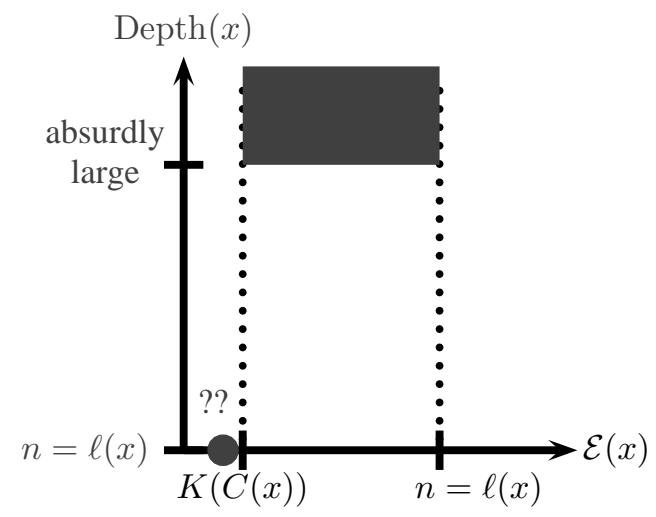

Fig. 2. At effective complexity equal to $K(C(x))$ logical depth suddenly becomes astronomically large. This is reminiscent of the phenomenon of "phase transition" known from statistical mechanics.

This theorem can easily be extended to the case of effective complexity with constraints as long as the constrained sets of ensembles satisfy the usual technical conditions:

Theorem 19 ( $\mathcal{E}$ and Depth with Constraints): There is a global constant $\omega \in \mathbb{N}$ with the following property: Suppose $f: \mathbb{N} \rightarrow \mathbb{N}$ is a strictly increasing, computable function. Furthermore, suppose that $x$ is a $k$-well behaved string, and the constrained set $\mathcal{C}$ is decidable and convex and contains the Dirac measure $\delta_{x}$. If the effective complexity of $x$ satisfies

$$
\mathcal{E}_{\delta, \Delta}(x \mid \mathcal{C})>K(C(x))+K(z)+K(f)+K(\mathcal{C})+\omega
$$

for some $\Delta \geq k+z+K(z)+K(f)+\omega+1+K(\mathcal{C})$ with $\delta \geq 0$ and $z \in \mathbb{N}$, then

$$
\operatorname{Depth}_{z}(x)>f(C(x)) .
$$

Remark. For an explanation and interpretation of this theorem, see the remarks after Theorem 18 above. 
Proof: The proof is almost identical to that of Theorem 18 above. The only modification is that the set $\tau_{y, f}$ has to be replaced by a set

$$
\begin{aligned}
\tau_{y, f, \mathcal{C}}:= & \left\{x \in\{0,1\}^{*} \mid \exists p \in\{0,1\}^{*} \text { with } V(p)=x,\right. \\
& \left.\ell(p) \leq y, T(p) \leq f(y), \delta_{x} \text { satisfies } \mathcal{C}\right\} .
\end{aligned}
$$

The convexity condition then ensures that the uniform distribution on $\tau_{y, f, \mathcal{C}}$, called $\mathbb{E}_{y, f, \mathcal{C}}$, is contained in $\mathcal{C}$. This construction enlarges every additive constant by a term $K(\mathcal{C})$, i.e. the complexity of a computer program that is able to test for strings if the corresponding Dirac measures are contained in $\mathcal{C}$.

\section{EFFECTIVE COMPLEXITY AND KOLMOGOROV Minimal Sufficient STATISTICS}

Now we study the relation between effective complexity without constraints and Kolmogorov minimal sufficient statistics (KMSS). For more information on Kolmogorov minimal sufficient statistics and related notions, see [1, 2.2.2].

For strings $x$ and integers $k \in \mathbb{N}$, we can define a (version of) the Kolmogorov structure function $H_{k}(x \mid n)$ by

$$
\begin{gathered}
H_{k}(x \mid n):=\min \left\{\log \# A \mid A \subseteq\{0,1\}^{n}, x \in A,\right. \\
\left.K_{*}(A \mid n) \leq k\right\},
\end{gathered}
$$

i.e. $H_{k}(x \mid n)$ is the logarithm of the minimal size of any subset of strings of length $n$ which contains the string $x$ and has complexity upper bounded by $k$, given $n$. The corresponding minimal set will be called $A_{k}$ (if there are several minimizers, we take the first set in some canonical order). Hence $H_{k}(x \mid n)=\log \# A_{k}$ and $K_{*}\left(A_{k} \mid n\right) \leq k$.

Definition 20 (KMSS):

Let $x$ be a string of length $n$ and denote by $k_{\Delta}(x)$ the minimal natural number $k$ satisfying

$$
H_{k}(x \mid n)+k \leq K_{*}(x \mid n)+\Delta .
$$

A minimal program $k_{\Delta}^{*}(x)$ for $A_{k_{\Delta}(x)}$ is called Kolmogorov minimal sufficient statistics of the string $x$.

Originally, the Kolmogorov structure function as well as Kolmogorov minimal sufficient statistics were defined by using plain Kolmogorov conditional complexity $C(\cdot \cdot \cdot)$ in (27) and (28) instead of Chaitin's prefix version $K_{*}(\cdot \mid \cdot)$. It holds

$$
\ell\left(k_{\Delta}^{*}(x)\right)=K\left(A_{k_{\Delta}(x)}\right) \stackrel{+}{<} k_{\Delta}(x)+K(n) .
$$

Moreover, $k_{\Delta}(x)$ can equivalently be written as

$$
\begin{aligned}
k_{\Delta}(x)=\min \left\{K_{*}(A \mid n) \mid\right. & \log \# A+K_{*}(A \mid n) \leq K_{*}(x \mid n) \\
& \left.+\Delta, \quad x \in A \subset\{0,1\}^{n}\right\} . \quad
\end{aligned}
$$

This formula looks very similar to the definition of (unconstrained) effective complexity, as given in Definition 4. Hence the Kolmogorov minimal sufficient statistics is approximately the minimal program of the minimizing set within the minimization domain of effective complexity. We will explore this observation in more detail in the following lemma.

Lemma 21: There is a constant $c \in \mathbb{N}$ such that for all $\delta, \Delta \geq 0$ it holds

$$
\mathcal{E}_{\delta, \Delta+c}(x) \stackrel{+}{<} \ell\left(k_{\Delta}^{*}(x)\right) \stackrel{+}{<} k_{\Delta}(x)+K(n)
$$

uniformly in $x \in\{0,1\}^{*}$, where $n:=\ell(x)$. Moreover, there is a constant $g \in \mathbb{N}$ such that for all $\delta, \Delta \geq 0$

$$
\begin{aligned}
\mathcal{E}_{\delta, \Delta}(x) \stackrel{+}{>} & k_{K(n)+\Delta+\delta(K(x)+\Delta)+K(\delta)+g}(x)-K(\delta) \\
\stackrel{+}{>} \ell\left(k_{K(n)+\Delta+\delta(K(x)+\Delta)+K(\delta)+g}^{*}(x)\right) & -K(\delta)-K(n)
\end{aligned}
$$

uniformly in $x \in\{0,1\}^{*}$.

Proof: Let $k:=k_{\Delta}(x)$ and $n:=\ell(x)$. By definition,

$$
k \geq K_{*}\left(A_{k} \mid n\right) \stackrel{ \pm}{=} K\left(A_{k}, n\right)-K(n) \stackrel{ \pm}{=} K\left(A_{k}\right)-K(n) .
$$

Let $\mathbb{E}_{k}$ be the uniform distribution on $A_{k}$. It follows

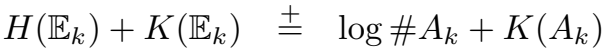

$$
\begin{aligned}
& =H_{k}(x \mid n)+K\left(A_{k}\right) \\
& \stackrel{+}{<} H_{k}(x \mid n)+k+K(n) \\
& \leq K_{*}(x \mid n)+\Delta+K(n) \\
& \stackrel{ \pm}{=} K(x, n)+\Delta \\
& \pm K(x)+\Delta \text {. }
\end{aligned}
$$

Thus, there is some constant $c \in \mathbb{N}$ such that

$$
\mathcal{E}_{\delta, \Delta+c}(x) \leq K\left(\mathbb{E}_{k}\right) \stackrel{+}{<} K\left(A_{k}\right) .
$$

Then (31) follows from (29).

In order to show (32), we use Lemma 13 Let $\mathbb{E}$ be the minimizing ensemble in the definition of $\mathcal{E}_{\delta, \Delta}(x)$. In particular, it holds

$$
K(\mathbb{E})=\mathcal{E}_{\delta, \Delta}(x) .
$$

Fix any $\varepsilon>0$. Due to Lemma 13 , there is a set $S^{\prime} \subset\{0,1\}^{*}$ containing $x$ such that

$$
\begin{aligned}
\log \# S^{\prime} & \stackrel{+}{<} H(\mathbb{E})(1+\delta), \\
K\left(S^{\prime}\right) & \stackrel{+}{<} K(\mathbb{E})+K(\delta) .
\end{aligned}
$$

Let now $S:=S^{\prime} \cap\{0,1\}^{n}$. It still holds $\log \# S \stackrel{+}{<} H(\mathbb{E})(1+\delta)$ and $K(S) \stackrel{+}{<} K\left(S^{\prime}\right)+K(n) \stackrel{+}{<} K(\mathbb{E})+K(\delta)+K(n)$. Since $K_{*}(S \mid n) \stackrel{ \pm}{=} K(S, n)-K(n) \stackrel{ \pm}{=} K(S)-K(n)$, we get the chain of inequalities

$$
\begin{aligned}
\log \# S+K_{*}(S \mid n) & +H(\mathbb{E})(1+\delta)+K(S)-K(n) \\
& +H(\mathbb{E})+\delta H(\mathbb{E})+K(\mathbb{E})+K(\delta) \\
\leq & K(x)+\Delta+\delta H(\mathbb{E})+K(\delta) \\
& + \\
& K_{*}(x \mid n)+K(n)+\Delta+K(\delta) \\
& +\delta(K(x)+\Delta) .
\end{aligned}
$$

Using (30) and (33), it follows that

$$
\begin{aligned}
k_{K(n)+\Delta+\delta(K(x)+\Delta)+K(\delta)+g}(x) & \leq K_{*}(S \mid n) \\
& \pm K(S)-K(n) \\
& \pm K(\mathbb{E})+K(\delta) \\
& =\mathcal{E}_{\delta, \Delta}(x)+K(\delta) .
\end{aligned}
$$

Then (32) follows again from (29) 


\section{CONCLUSIONS}

We have given a formal definition of effective complexity and rigorous proofs of its basic properties. In particular, we have shown that there is an interesting relation between effective complexity and logical depth: the depth of a string $x$ is astronomically large if the effective complexity exceeds $K(C(x))$; otherwise, it can be arbitrarily small.

This statement is true up to a few technical conditions and up to certain additive constants. These constants become less and less important for longer and longer strings - this is comparable to the "thermodynamic limit" in statistical mechanics, and the behavior can be compared to that of a phase transition.

So how useful is effective complexity for the study of natural systems? We do not yet know the answer to this question, but we hope that our mathematically rigorous approach gives the first steps towards possible applications within mathematics or theoretical computer science.

At least, we have shown that effective complexity has interesting properties, for example there are strings that have effective complexity close to their lengths. Those strings are rare events only - "most" strings are in fact effectively simple; this follows from Theorem 10. But this property is unavoidable: Recall that a major motivation for the definition of effective complexity was that random strings should be simple (in contrast to Kolmogorov complexity). Now since almost all strings are random, it follows that almost all strings must be effectively simple.

Most of our results concerning the constrained version of effective complexity $\mathcal{E}_{\delta, \Delta}(x \mid \mathcal{C})$ were derived under the assumption that the Dirac measure $\delta_{x}$ is an element of $\mathcal{C}$. Although this is a natural assumption, the behavior of effective complexity might as well be completely different if it is dropped. Investigating such situations in more detail could be useful in order to get better insight into the concept of effective complexity and its limitations.

Finally, a possible field of application of effective complexity might be statistical mechanics, where the notion of entropy and algorithmic complexity have both already led to interesting conclusions. After all, the constraints given by the set $\mathcal{C}$ can be interpreted as macroscopic observables as discussed in Section [II] and we have already compared our result on logical depth with certain notions of statistical mechanics.

\section{APPENDIX A}

\section{An EXAMPle of Non-COMPUTABle ENTROPY}

As promised in the introduction, we give an explicit construction of a computable ensemble $\mathbb{E}$ with the property that the entropy $H(\mathbb{E})$ is finite but not computable:

Example 22 (Non-Computable Entropy):

For every $n \in \mathbb{N}$, let $A_{n}$ be the set of strings that start with exactly $n-1$ zeroes, such that the $n$-th bit either does not exists or is a one. That is,

$$
\begin{aligned}
& A_{1}=\{\lambda, 1,10,11,100,101, \ldots\}, \\
& A_{2}=\{0,01,010,011,0100,0101, \ldots\}, \\
& A_{3}=\{00,001,0010,0011,00100,00101, \ldots\}
\end{aligned}
$$

and so on. Clearly, this is a computable partition of $\{0,1\}^{*}$ into countably-infinite, mutually disjoint subsets: $\bigcup_{n \in \mathbb{N}} A_{n}=$ $\{0,1\}^{*}, A_{m} \cap A_{n}=\emptyset$ if $m \neq n$.

We now construct an ensemble $\mathbb{E}$ such that every set $A_{n}$ has weight $2^{-n}$, that is $\mathbb{E}\left(A_{n}\right):=\sum_{x \in A_{n}} \mathbb{E}(x)=2^{-n}$. We distribute the weight $2^{-n}$ among the members of $A_{n}$ in a way, such that the resulting ensemble $\mathbb{E}$ is computable, but has noncomputable entropy. This is done as follows: let $\Omega>0$ be a real number which is not computable, but enumerable from below. That is, there exists a computable sequence $\left(\Omega_{n}\right)_{n \in \mathbb{N}}$ with $\Omega_{1}:=0$ which is increasing, i.e. $\Omega_{n+1} \geq \Omega_{n}$, and which converges to $\Omega$, i.e. $\lim _{n \rightarrow \infty} \Omega_{n}=\Omega$. For example, we may use Chaitin's Omega number[1]

$$
\Omega:=\sum_{U(x) \text { exists }} 2^{-\ell(x)},
$$

where the sum is over all strings $x \in\{0,1\}^{*}$ such that the universal prefix computer $U$ halts on input $x$. The number $\Omega$ gives the probability that the computer $U$ halts on randomly chosen input. It is a real number between zero and one, and it is obviously enumerable from below, but it is not computable.

Given some weight $c \in(0,1]$ and finitely many positive real numbers $r_{i}$ such that $\sum_{i} r_{i}=c$, the resulting entropy sum $-\sum_{i} r_{i} \log r_{i}$ will always be larger than or equal to $-c \log c$. The converse is also true: Given some fixed entropy value $s \geq-c \log c$, we can always find finitely many positive real numbers $r_{i}$ with $\sum_{i} r_{i}=c$ such that $-\sum_{i} r_{i} \log r_{i}=s$. Such a list of real numbers can be found in an obvious systematic way that can be implemented as a computer program.

Thus, to every $n \in \mathbb{N}$, we may systematically distribute the weight $2^{-n}$ to finitely many strings in $\left\{x_{1}, \ldots, x_{k}\right\} \subset A_{n}$ such that the corresponding probabilities $\mathbb{E}\left(x_{i}\right)$ have entropy sum $-2^{-n} \log 2^{-n}+\Omega_{n+1}-\Omega_{n}$, i.e.

$$
\begin{aligned}
\sum_{x \in A_{n}} \mathbb{E}(x) & =2^{-n}, \\
-\sum_{x \in A_{n}} \mathbb{E}(x) \log \mathbb{E}(x) & =-2^{-n} \log 2^{-n}+\underbrace{\Omega_{n+1}-\Omega_{n}}_{\geq 0} .
\end{aligned}
$$

The resulting ensemble is obviously computable, and the entropy is

$$
\begin{aligned}
H(\mathbb{E}) & =-\sum_{n=1}^{\infty} \sum_{x \in A_{n}} \mathbb{E}(x) \log \mathbb{E}(x) \\
& =\sum_{n=1}^{\infty}\left(-2^{-n} \log 2^{-n}+\Omega_{n+1}-\Omega_{n}\right) \\
& =\sum_{n=1}^{\infty} \frac{n}{2^{n}}+\lim _{N \rightarrow \infty} \Omega(N)-\Omega(1) \\
& =2+\Omega .
\end{aligned}
$$

This is not a computable number.

\section{ACKNOWLEDGMENT}

The authors would like to thank Eric Smith for helpful discussions. This work has been supported by the Santa $\mathrm{Fe}$ Institute. 


\section{REFERENCES}

[1] M. Li and P. Vitanyi, An Introduction to Kolmogorov Complexity and Its Applications, Springer Verlag (1997)

[2] M. Gell-Mann, S. Lloyd, Effective Complexity, Santa Fe Institute, pp. 387-398 (2003)

[3] M. Gell-Mann, S. Lloyd, Information Measures, Effective Complexity, and Total Information, Complexity, Vol. 2, 44-52 (1996)

[4] C. Bennett, Logical Depth and Physical Complexity, in The Universal Turing Machine - a Half-Century Survey, ed. Rolf Herken, Oxford University Press (1988)

[5] S. Lloyd, Measures of Complexity: A Nonexhaustive List, IEEE Control Systems Magazine 21/4, pp. 7-8 (2001)

[6] P. Gács, J. T. Tromp, P. M. B. Vitányi, Algorithmic Statistics, IEEE Trans. Inf. Th. 47/6 2443-2463 (2001)

[7] G.J. Chaitin, Exploring Randomness, Springer-Verlag London (2001)

[8] T. M. Cover, J. A. Thomas, Elements of Information Theory, Wiley Series in Telecommunications, John Wiley \& Sons, New York (1991)

[9] N. Ay, M. Müller, A. Szkoła, Effective Complexity of Ergodic Processes (preliminary title), in preparation. 